\title{
Cluster-based Access Control Mechanism for Cellular D2D Communication Networks with Dense Device Deployment
}

\author{
Thanh-Dat Do ${ }^{1}$, Ngoc-Tan Nguyen*2, Thi-Huong-Giang Dang ${ }^{3}$, Nam-Hoang Nguyen ${ }^{4}$, Minh-Trien Pham ${ }^{5}$ \\ VNU University of Engineering and Technology, Vietnam National University, Hanoi, Vietnam ${ }^{1,3,4,5}$ \\ Thang Long University, Hanoi, Vietnam ${ }^{2}$ \\ University of Economics-Technique and Industry, Hanoi, Vietnam ${ }^{3}$
}

\begin{abstract}
In cellular device-to-device (D2D) communication networks, devices can communicate directly with each other without passing through base stations. Access control is an important function of radio resource management which aims to reduce frequency collision and mitigate interference between user's connections. In this paper, we propose a cluster-based access control (CBAC) mechanism for heterogeneous cellular D2D communication networks with dense device deployment where both the macro base station and smallcell base stations (SBSs) coexist. In the proposed CBAC mechanism, relied on monitoring interference from its neighboring SBSs, each SBS firstly selects their operating bandwidth parts. Then, it jointly allocates channels and assigns transmission power to smallcell user equipments (SUEs) for their uplink transmissions and users using D2D communications to mitigate their interference to uplink transmissions of macrocell user equipments (MUEs). Through computer simulations, numerical results show that the proposed CBAC mechanism can provide higher network throughput as well as user throughput than those of the network-assisted devicedecided scheme proposed in the literature. Simulation results also show that SINR of uplink transmissions of MUEs and D2D communications managed by the MBS can be significantly improved.
\end{abstract}

Keywords-D2D communications; access control; channel allocation; power assignment; interference mitigation

\section{INTRODUCTION}

Future mobile networks are expected to provide communication services to billions of user equipments (UEs), i.e., regular mobile users and machine-type communication devices. In these networks, devices require a huge wireless traffic demand of device-to-device (D2D) communications such as vehicle to vehicle communications, communications between IoT devices. In traditional cellular networks, a base station (BS) acts as a relay to provide D2D communications for its users. Recently, cellular networks with D2D communications allow two arbitrary devices to directly establish a D2D communications link. With the aid of D2D communications, these networks can obtain significant improvements in terms of spectrum reuse, traffic offloading, low latency, and system throughput [1]-[4]. Nonetheless, cellular networks with D2D communications also bring lots of technical challenges such as high signaling load and frequency collisions (which may cause the degradation of the signal-to-interference-plus-noise ratio (SINR)).

\footnotetext{
*Corresponding Author: Ngoc-Tan Nguyen
}

In cellular networks with D2D communications, there are two type of communications, i.e., conventional cellular communications between BSs and their UEs, and D2D communications between two UEs. In the inband-overlay mode, different frequency bands are allocated for cellular and D2D communications, thus D2D communications cannot cause interference to cellular communications. Thus, the quality of service (QoS) of cellular communications is not affected by D2D communications but the efficiency of spectrum utilization is typically low [4]. By contrast, in the inband-underlay mode, a same frequency spectrum is allocated for both cellular and D2D communications. In this mode, the signals of a D2D communications might cause D2D-to-cellular interference to cellular communications when they use same channels [5][8], [9]. It is worth noting that cellular communications are given higher priority than $\mathrm{D} 2 \mathrm{D}$ communications. To mitigate D2D-to-cellular interference, an efficient access control mechanism including channel allocation and transmission power assignment is needed to handle D2D connection requests.

Channel allocation, transmission power assignment, and interference mitigation are crucial research issues in cellular D2D communications networks. Power control can be implemented in different approaches, i.e., centralized manner or distributed manner [10]-[11]. In [10], the authors show that power control using the centralized algorithm can obtain higher performance than that using the distributed algorithm, but it suffers higher overhead as the number of devices increases. By contrast, in the distributed algorithm, D2D users exploit local channel state information to decide the transmission power, thus the overhead can be reduced. However, they might use high transmission power which can cause high interference to cellular users. Similar results for both centralized and distributed algorithms are also presented in the work [11]. The work in [12] provides a survey of radio resource management methods to reduce interference between D2D users and cellular users. Channel reuse technique using orthogonal frequency-division multiplexing is proposed in [13] where D2D and cellular users can share same spectrum. In the work [14], a joint admission control and resource allocation strategy is proposed to provide QoS support to cellular and D2D communications. The authors in [15] propose a resource scheduling method based on user location. However, in dense device networks, processing information of users' locations might cause high computational load. A guard zone based D2D-activation scheme is proposed in [16] in which the 


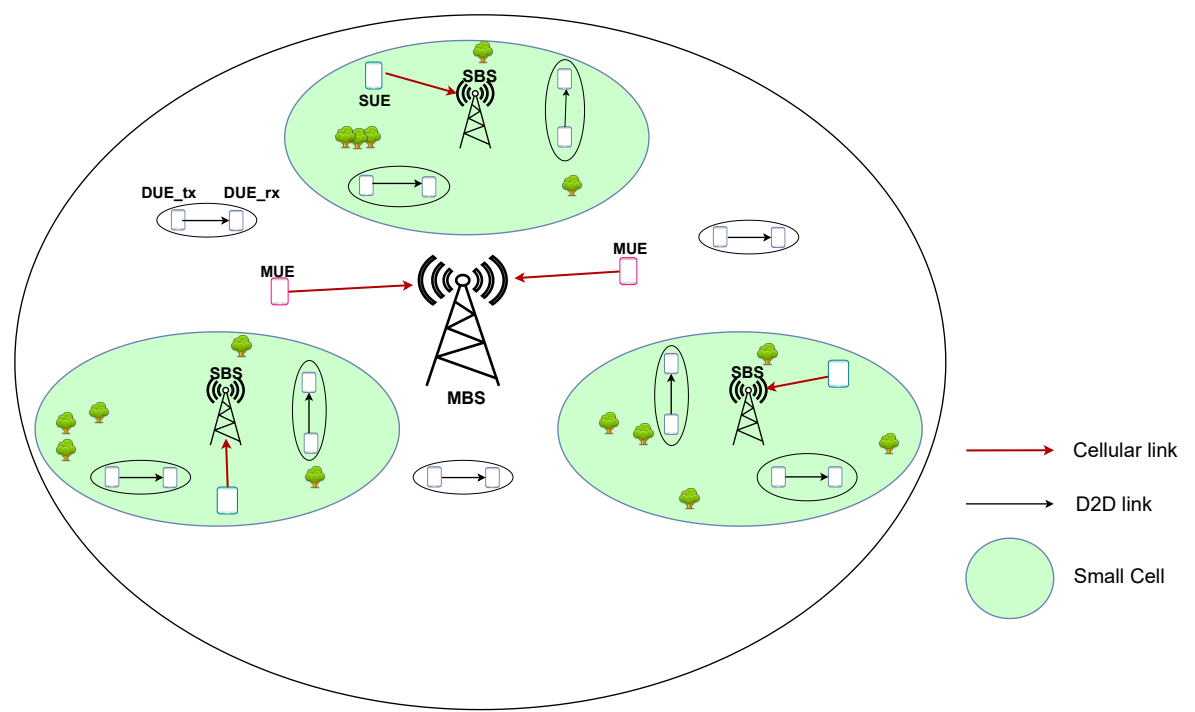

Fig. 1. A Dense Heterogeneous Cellular Network with D2D Communications.

exact closed-form expressions for the successful transmission probability of cellular users are proposed under the assumption that D2D users are uniform distributed within a geographical area. The scheme optimizes the guard zone's inner radius under the criteria of maximizing both transmission power and average throughput. Yet, this approach is only efficient for mobile networks with low user density.

The aforementioned works only study separated problems of channel allocation and power control which might lower the system performance. The authors in [9] analyze the impacts of co-channel interference between D2D links. Co-channel interference is unavoidable when the device density is ultra high. By joint optimizing channel allocation and power control, the interference mitigation efficiency and system performance can be significantly improved. A two-stage energy efficient maximization method including the power control and the channel allocation algorithms to improve D2D pair energy efficiency is proposed in [17] for D2D networks with low density. For dense D2D communication networks, the proposed method might cause high computation load. In [18], a distributed channel allocation and power control method based on Stackelberg game for D2D underlaid cellular networks is proposed to improve the sum-rate of D2D communications while meeting the QoS requirements of cellular users. This method can reduce the computation load of the base station effectively in small D2D communication networks. In [19], the authors propose a centralized resource management mechanism including channel allocation and transmission power control for heterogeneous cellular networks assisted by D2D communications. This mechanism can significantly improve the system throughput by mitigating D2D-to-cellular interference. However, it requires high computation load of the MBS and the channel measurement capability of UEs as the numbers of UEs and channels increase.

To our best knowledge, a practical access control mechanism for dense heterogeneous cellular networks with D2D communication assistance in which both the MBS and smallcell base stations (SBSs) coexist has not been investigated in the literature. In this paper, new constraints including the dense deployment of UEs and SBSs, flexible spectrum management (i.e., allocating multi bandwidth parts (BWPs) for UEs), and signaling load requirements are considered for the proposed heterogeneous cellular network assisted by D2D communications. Then, we propose a cluster-based access control mechanism involving the BWP selection for SBSs, channel allocation, and power assignment to smallcell user equipments and users using D2D communications to mitigate D2D-tocellular interference as well as enhance network throughput.

The remainder of the paper is organized as follows. The system model of the proposed heterogeneous cellular D2D communication networks with dense device deployment is described in Section 2. Section 3 presents the proposed clusterbased access control mechanism. Simulation results and discussions are provided in Section 4. Finally, the conclusion is presented in Section 5.

\section{SYSTEM MODEL}

\section{A. System Model}

As illustrated in Fig. 1, the proposed system model consists of a macro base station (MBS) and $S$ smallcell base stations (SBSs) located randomly in the coverage area of the MBS to increase the network capacity. There are three considered types of user equipments (UEs): (1) macrocell UEs (MUEs) served by the MBS, (2) smallcell UEs (SUEs) served by the according SBS, (3) users using D2D communications (DUEs). Under this setting, D2D communications allow the DUEs to exchange their data to each other directly to provide low latency communications. The coverage area of MBS is divided to multiple sectors in which the uplink transmissions to the MBS and SBSs, and D2D communications share a same frequency spectrum of $N_{C}$ channels. This spectrum is divided to $M$ bandwidth parts (BWPs). Each uplink transmissions to the MBS and SBSs utilizes one channel belonging to a BWP. A D2D communication between a pair of DUEs is also allocated one channel of a BWP. Each BWP has $N_{C}^{B W P}$ channels for data transmissions and one reference signal (RS) channel for 
TABLE I. Matching TABle Between the SPectrum EFFiciency and SINR [19]

\begin{tabular}{|c|c|c|c|}
\hline Modulation & $\begin{array}{c}\text { Code Rate (Default } \\
\text { Repetition=1) }\end{array}$ & $\begin{array}{c}\text { Spectrum Efficiency } \eta \\
(\mathbf{b p s} / \mathbf{H z})\end{array}$ & Minimum SINR (dB) \\
\hline \hline QPSK & $1 / 2(4)$ & 0.25 & -2.5 \\
\hline QPSK & $1 / 2(2)$ & 0.5 & 0.5 \\
\hline QPSK & $1 / 2$ & 1 & 3.5 \\
\hline QPSK & $3 / 4$ & 1.5 & 6.5 \\
\hline $16-$ QAM & $1 / 2$ & 2 & 9 \\
\hline $16-$ QAM & $3 / 4$ & 3 & 12.5 \\
\hline $64-$ QAM & $1 / 2$ & 3 & 14.5 \\
\hline 64-QAM & $2 / 3$ & 4 & 18.5 \\
\hline 64-QAM & $3 / 4$ & 4.5 & \\
\hline
\end{tabular}

broadcasting the RS. When a SBS uses a BWP, the SBS broadcasts the predefined RS on its RS channel of the BWP at a fixed transmission power. A SBS forms a D2D cluster and is allowed to use up to $m$ BWPs of the total $M$ BWPs of the network. Among of them, $m_{S U E}$ BWPs can be used for uplink transmissions of SUEs and $m_{D 2 D}$ BWPs can be allocated to D2D communications.

\section{B. Pathloss Models and Interference Analysis}

In the literature, various channel models are considered for the D2D communication networks [19]-[22]. In this paper, channel models proposed in [19] are adopted for the performance comparison. The channel model includes two transmission modes, i.e., Line-of-Sight (LOS) and non-Lightof-Sight (NLOS). The pathloss of these transmission modes are estimated as follows:

- The LOS pathloss model is applied to calculate the pathloss between the MBS and MUEs, the MBS and its DUEs, a SBS and its SUEs, and a SBS and its DUEs. The LOS pathloss is calculated as follows:

$$
P L(d)=127+30 \log _{10}(d)+\varsigma .
$$

- The NLOS pathloss model is applied to the D2D communications and uplink channels between DUEs and MUEs, DUEs and SUEs, and MUEs and SUEs. The NLOS pathloss is calculated as follows:

$$
P L(d)=128.1+37.6 \log _{10}(d)+\varsigma,
$$

where $d$ is the distance between a sender and a receiver in kilometers. $\varsigma$ is the shadowing in $\mathrm{dB}$ which follows log-normal distribution with the mean is zero and the standard deviation is one.

The total throughput obtained by a UE (i.e., a MUE, SUE, or DUE) is calculated as follows:

$$
C=\sum_{i=1}^{N_{c h}} \eta_{i} B_{i},
$$

where $N_{c h}$ is the number of channels used by the UEs. $B_{i}$ denotes the bandwidth of the channel $i$-th among $N_{c h}$ channels. The spectrum efficiency of the channel $i$-th which (denoted as $\eta_{i}$ ) depends on the SINR measured at the receiver (as shown in Table I [19]). To increase the spectrum efficiency of the proposed system, channels are reused in both D2D communications and uplink transmissions in the MBS and SBSs. However, this leads to the co-channel interference to the MUE. Specifically, when a SBS allocate a channel which is using by the MUE to its SUEs or DUEs, this can cause cochannel interference to the MUE. The D2D communications managed by the MBS can also interfere the uplink transmission of the MUE if they use same channel. Therefore, there is a need of an access control mechanism to allocate channel and optimize transmission power to minimize the co-channel interference.

\section{Cluster-Based Access Control Mechanism}

In order to mitigate co-channel interference as well as improve the system throghput, in this section, a cluster-based access control (CBAC) mechanism, which consists of channel allocation and power control, is designed for both the MBS and SBSs in the proposed D2D mobile network with the dense deployment of SBSs and DUEs. Firstly, a SBS selects its BWPs and forms its cluster (involving its SUEs and DUEs). The SBS can accept or remove a UE (i.e. a SUE or DUE) when the UE enters or moves out its coverage area, respectively. Then, the SBS performs the proposed CBAC mechanism to SUEs and DUEs those are in its cluster. While, the MBS performs the proposed CBAC mechanism to its MUEs and DUEs those do not belong to any cluster.

The functions of the MBS and SBSs are listed in detail as follows:

\section{A. A SBS Selects BWPS}

1) When a SBS configures its operating BWPs, it measures energy levels of RS channels of $M$ BWPs, and then selects $m$ BWPs having the lowest energy levels among $M$ BWPs.

2) Then, among $m$ selected BWPs, it assigns $m_{D 2 D}$ BWPs for D2D communications which have lower RS energy levels than those of the remainder $\left(m_{S U E}\right)$ BWPs for cellular communications.

3) Finally, it informs the MBS about its BWP configuration. The MBS is then responsible to update the number of SBSs using the same BWP.

\section{B. A SBS Manages its Cluster and Estimate the Maximum Acceptable Interference}

1) Accept a new UE: The SBS periodically broadcasts its pilot signal. If a new UE wants to be served by an 
SBS, it detects a SBS with the strongest pilot signal among all SBSs in its range. Then, it sends a request to that SBS for cluster registration. If the SBS still has an available room for the UE, it accepts the UE to join its cluster. Otherwise, the SBS rejects and informs the UE to look for another available SBS.

2) Remove an inactive UE: A UE might be inactive when it is off or moves out the coverage area of its serving SBS. The serving SBS periodically asks its UEs to confirm whether they are still active or not. If the serving SBS does not receive any confirmation from a UE, the serving SBS can remove the UE out of its cluster.

3) The SBS estimates the maximum acceptable interference: It is worth noting that the channel allocated to a D2D communication or an uplink cellular transmission to the SBS might be also used by a MUE. In that case, the SUEs or DUEs (served by the SBS and MBS) can cause interference to the MUE. Therefore, the SBS must control the transmission power of its SUE and source DUE subject to their interference to the MUE that does not exceed the maximum acceptance interference. It is worth noting that the worst case of an edge MUE which is most vulnerable to interference from SUEs and DUEs is considered. Firstly, the MBS informs the SBS about the maximum acceptable interference $I_{\text {total }}^{\max }$ that the edge MUE still can guarantee its SINR threshold:

$$
I_{\text {total }}^{\max }=\frac{P_{\max }^{M U E}}{\gamma^{0} P L(R)},
$$

where $P_{\max }^{M U E}$ is the maximum transmission power of the MUE. $P L(R)$ is the estimated pathloss of the channel link from the edge MUE to the MBS with $R$ is the radius of the MBS's cell. $\gamma^{0}$ denotes the SINR threshold of the uplink transmission from the MUE to the MBS. Under the worst setting, all neighbors of the SBS also allocate the same channel to their SUEs or DUEs. Assume that the SBS can detect $N_{S B S}$ neighbors, then the SBS can calculate the maximum acceptable interference that its DUE or SUE can cause to the edge MUE:

$$
I_{S B S}^{\max }=\frac{I_{\text {total }}^{\max }}{N_{S B S}+1} .
$$

C. A SBS Allocates Channel And Assign Transmission Power To Its SUES

1) When a SUE wants to establish an uplink connection with its SBS, the SUE firstly sends a connection request to its $\mathrm{SBS}$.

2) Then, the serving SBS finds a BWP that has available channels and the lowest RS energy level among $m_{S U E}$ BWPs. If a qualified BWP is found, the SBS allocates a round-robin free channel of the BWP to the SUE with an uplink transmission power $P S B E$ assigned as follows:

$$
P_{S B S}^{S U E}=I_{S B S}^{\max } P L\left(d_{M B S \rightarrow S B S}\right),
$$

where $P L\left(d_{M B S \rightarrow S B S}\right)$ is the estimated pathloss from the MBS to the serving SBS. Assume that the pathloss information of SUEs is not available at the serving SBS (since it does not know the exact locations of SUEs). Thus, the pathloss from SUEs to the SBS is approximateed as the pathloss from the MBS to SBS.

\section{A SBS Allocate Channel And Assign Transmission Power To Its DUEs}

1) When a DUE wants to establish a D2D communication with another DUE, the DUE firstly sends a D2D connection request to its serving SBS.

2) Then, the serving SBS finds a BWP which has available channels and the lowest RS energy level among $m_{D 2 D}$ BWPs. If a qualified BWP is found, the SBS allocates a round-robin free channel of the BWP to the DUE with a transmission power $P_{S B S}^{D U E}$ assigned as follows:

$$
P_{S B S}^{D U E}=I_{S B S}^{\max } P L\left(d_{M B S \rightarrow S B S}\right)
$$

E. The MBS Assigns Channel and Transmission Power to its MUES

1) When a MUE wants to establish an uplink transmission to the MBS, the MUE firstly sends a connection request to the MBS.

2) Then, the MBS allocates a round-robin free channel to the MUE and assign an initial transmission power $P_{M B S}^{M U E}$ to the allocated channel.

3) After the uplink transmission between the MUE and the MBS is established, they collaborate to optimize the uplink transmission power for the MUE. Firstly, the MBS measures the SINR of the uplink channel and finds an optimal transmission power (that guarantees the SINR target) for the MUE. Then, it sends a power control message with the optimal transmission power information to the MUE.

F. The MBS Assigns a Available Channel and Transmission Power to DUEs that do not Belong to any Clusters

1) When a DUE, which is currently served by the MBS, wants to establish a D2D communication with another DUE, it sends a D2D connection request to the MBS. Then, the MBS allocates a round-robin free channel of its BWP to the DUE.

2) The worst case is considered in which all SBSs also allocate the same channel to its UEs that causes interference to the D2D communications served by the MBS. Since the MBS knows the number of SBSs (denoted as $N_{S B S}^{\prime}$ ) which are using the same BWP. Then, the MBS estimates the maximum acceptable interference $\left(I_{M B S}^{\max }\right)$ that the SBS's UEs and the DUE (served by the MBS) can cause to the MUE:

$$
I_{M B S}^{\max }=\frac{I_{\text {total }}^{\max }}{N_{S B S}^{\prime}+1}
$$

3) Finally, the MBS assigns the transmission power to the DUE:

$$
P_{M B S}^{D U E}=I_{M B S}^{\max } P L\left(d_{D U E \rightarrow M B S}\right),
$$

where $P L\left(d_{D U E \rightarrow M B S}\right)$ is the estimated pathloss from the DUE to the MBS. 


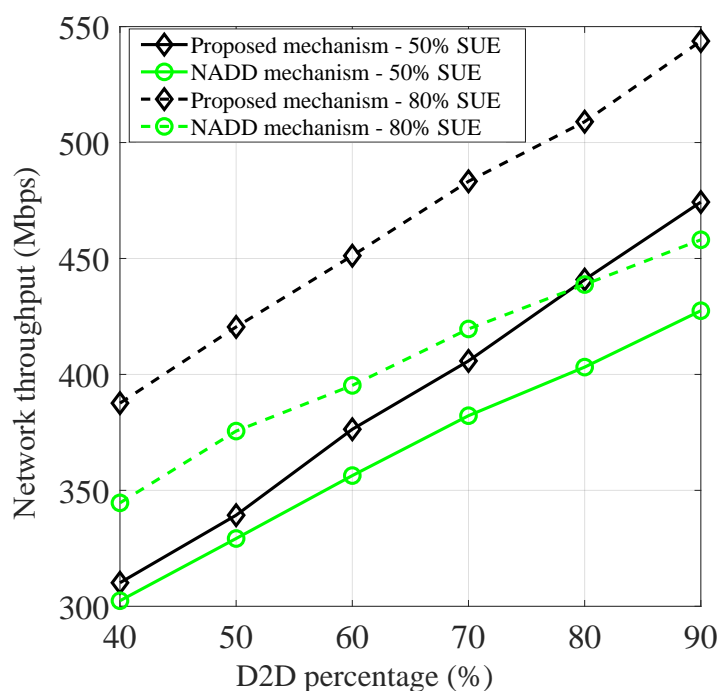

(a)

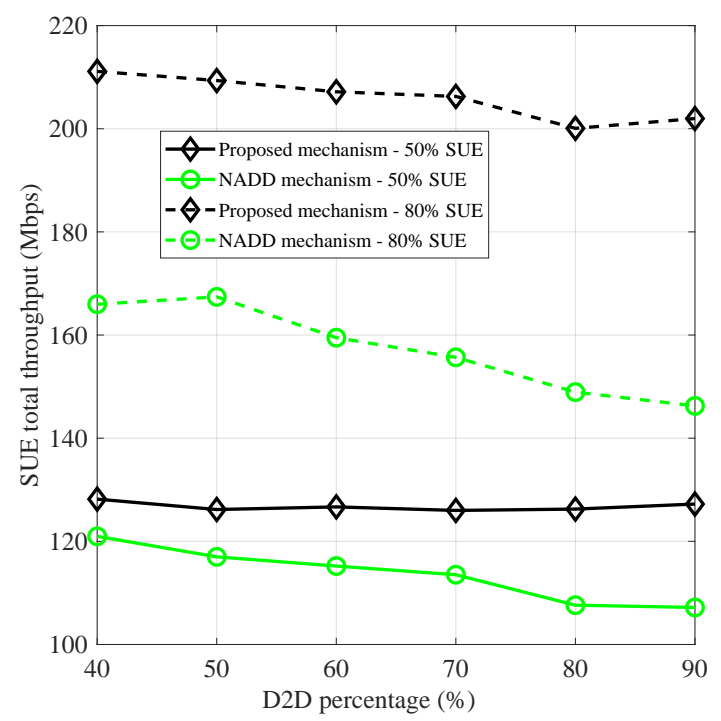

$(c)$

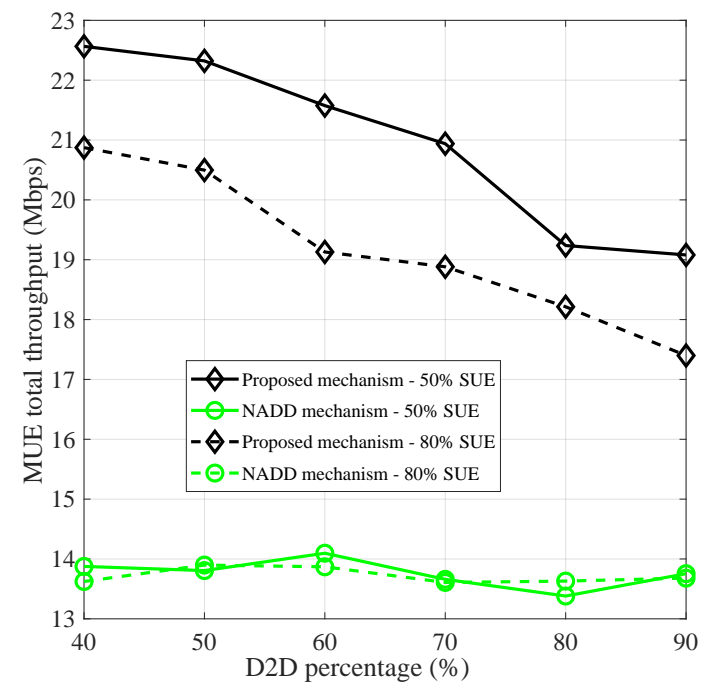

(b)

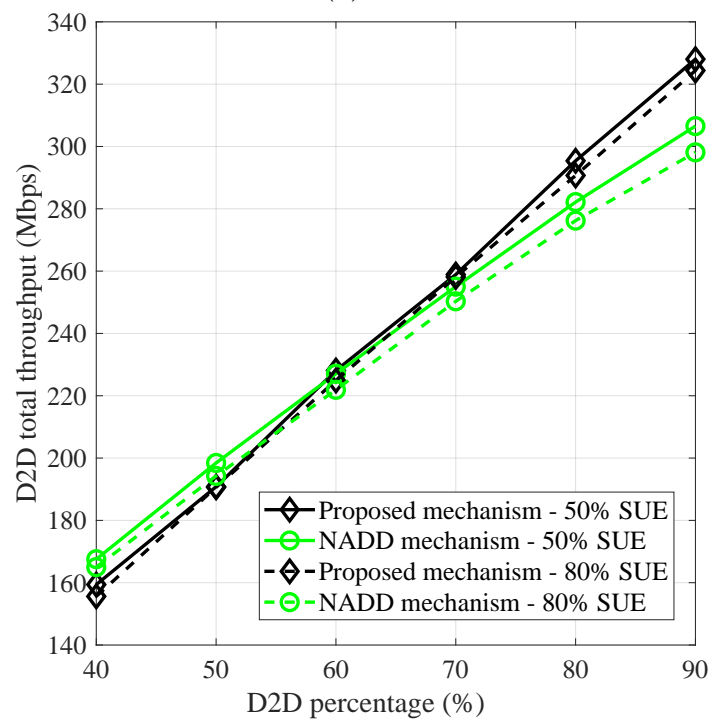

$(d)$

Fig. 2. (a) Network Throughput, (b) Total Throughput Obtained by MUEs, (c) Total Throughput Obtained by SUEs, (d) Total Throughput Obtained by D2D Communications vs. D2D Percentage.

\section{Simulations AND Numerical Results}

In the section, we conduct computer simulations and performance evaluations of the proposed cluster-based access control (CBAC) mechanism and the dynamic network assisted device decided (NADD) mechanism proposed in [19]. In our simulations, we consider a MBS and 50 SBSs under the coverage of the MBS (having a radius of $1000 \mathrm{~m}$ ). Each SBS has the coverage radius of $100 \mathrm{~m}$. The spectrum has 60 channels divided into 6 BWPs and each channel has the bandwidth of $180 \mathrm{KHz}$. Under this setting, each MUE or SUE uses one channel for their uplink transmissions and the other channel is used for D2D communications. The maximum transmission power of MUEs, SUEs, and DUEs is $23 \mathrm{dBm}$ [23]-[25]. Each SBS is assumed to consume two BWPs, the former is used for SUEs' uplink transmissions and the latter is used for D2D communications. The SUE and D2D percentages of a SBS are defined as the ratios of the number of simultaneous SUEs' uplink transmissions and D2D communications to the total number of available channels of the SBS, respectively. Other setting parameters are listed in Table II.

\section{A. Throughput Performance}

For throughput performance comparison, two scenarios, i.e., different D2D percentages and SINR thresholds, are investigated to evaluate the throughput performance.

1) Varying D2D Percentage: Fig. 2(a) - Fig. 2(d) show the comparisons of the network throughput, total throughput obtained by MUEs, SUEs, and DUEs (via D2D communications), respectively, when varying the D2D percentage. Two different 
TABLE II. Simulation PARAMETERS.

\begin{tabular}{|l|l|l|}
\hline \multicolumn{1}{|c|}{ Setting Parameters } & \multicolumn{1}{|c|}{ Value } & \multicolumn{1}{c|}{ Unit } \\
\hline \hline Macrocell radius (R) & 1000 & $\mathrm{~m}$ \\
\hline Number of channels & 60 & channel \\
\hline Number of BWPs & 6 & BWP \\
\hline Number of SBSs & 50 & SBS \\
\hline Number of BWPs in a SBS & 2 & BWP \\
\hline Smallcell radius & 100 & $\mathrm{~m}$ \\
\hline Bandwidth of a subchannel & 180 & $\mathrm{KHz}$ \\
\hline Number of macrocell UEs & 50 & MUE \\
\hline Number of SUEs in a SBS & 8 & SUE \\
\hline MUE channel usage & 1 & channel \\
\hline SUE channel usage & 1 & channel \\
\hline D2D channel usage & 1 & channel \\
\hline Maximum transmission power of MUE/device & 23 & $\mathrm{dBm}$ \\
\hline Mean distance between two devices in D2D pair & 20 & $\mathrm{~m}$ \\
\hline MUE's SINR target for power control & 20 & $\mathrm{~dB}$ \\
\hline MUE's SINR threshold & 7.5 & $\mathrm{~dB}$ \\
\hline Carrier frequency & 2.0 & $\mathrm{GHz}$ \\
\hline
\end{tabular}

settings of the SUE percentage in each SBS (i.e., $50 \%$ and $80 \%$ ) have been considered in all simulations. In Fig. 2(a), simulation results show that the proposed CBAC mechanism provides higher network throughput than that of the NADD mechanism. For example, when the SUE and D2D percentage are $50 \%$ and $70 \%$, respectively, the network throughput of the proposed CBAC mechanism is $10 \%$ higher than that of the NADD mechanism. As the D2D traffic load increases, the network throughput also increases since SBSs can accept any new D2D connection requests until all D2D channels of SBSs are occupied. However, when the D2D traffic load increases, the throughput obtained by MUEs and SUEs are decreased as shown in Fig. 2(b) and Fig. 2(c), respectively. It is due to the fact that D2D communications can cause interference to MUEs and SUEs. Thus, the more D2D communications, the higher interference to MUEs and SUEs. It is recommended that in the cellular D2D mobile network, it is necessary to set a limit on the number of D2D communications. As can be seen in Fig. 2(d), the total throughput obtained by D2D communications of the proposed CBAC mechanism is higher than that of the NADD mechanism. The reason is that in the proposed CBAC mechanism, a SBS can select BWPs with low interference levels for D2D communications which results in lower interference from other SBSs and MUEs.

2) Varying SINR threshold: Fig. 3 illustrates the total throughput obtained by MUEs, SUEs and D2D communications as varying the SINR threshold of the MUE under the setting of the SUE and D2D percentages at $80 \%$. When the SINR threshold of the MUE increases, the maximum acceptable interference is decreased which results in reducing the transmission power of SUEs and DUEs. Therefore, as shown in Fig. 3, the total throughput obtained by MUEs is increased. Overall, the proposed CBAC mechanism has higher total throughput obtained by MUEs, SUEs and D2D communications than those of the NADD mechanism. The reason is that the proposed CBAC mechanism is able to avoid the frequency collision between uplink transmissions of SUEs and D2D communications, thus reduce the co-channel interference from SUEs and D2D communications to MUEs.

\section{B. Interference Mitigation}

To evaluate the interference mitigation of the proposed CBAC mechanism for co-channel interference from uplink

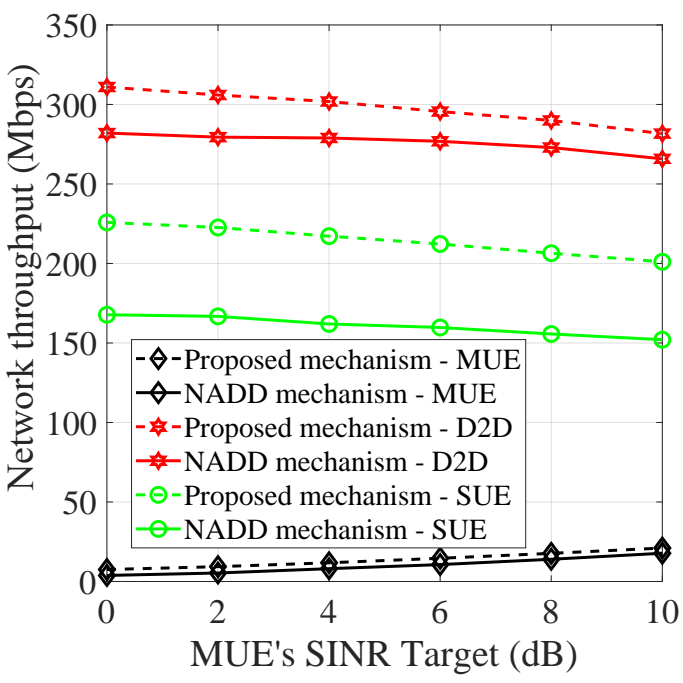

Fig. 3. Network Throughput vs MUE's SINR Threshold.

transmissions of SUEs and D2D communications to MUEs, we plot the statistical cumulative distribution functions (CDFs) w.r.t. SINRs of received signals of the MUEs' and SUEs' uplink transmissions, and D2D communications, respectively, which are measured at the MBS when the SUE percentage of each SBS is $80 \%$. As shown in Fig. 4(a), at the SINR value of $7 \mathrm{~dB}$, the NADD mechanism can provide $30 \%$ of SINR samples less than $7 \mathrm{~dB}$ whereas the proposed CBAC mechanism provides $40 \%$ of SINR samples less than $7 \mathrm{~dB}$. However, at the higher SINR value, e.g., 10dB, the proposed and NADD mechanisms provide about $45 \%$ and $90 \%$ of SINR samples less than $10 \mathrm{~dB}$, respectively. That means the proposed CBAC mechanism is able to mitigate the interference from SUEs and D2D communications to MUEs in the case of dense device deployment. Fig. 4(b) and Fig. 4(c) also show that the proposed CBAC mechanism provides better SINR than the NADD mechanism. It is due to the fact that in the proposed CBAC mechanism, each SBS forms a cluster and allocates different BWPs to SUEs' uplink transmissions and D2D communications in its cluster, thus the interference between SUEs' uplink transmissions and D2D communications are locally eliminated which results in the improvement of SINR of SUEs and D2D communications.

\section{CONClusion}

In this paper, we have studied a heterogeneous cellular D2D communication networks with new constraints of dense device deployment, flexible spectrum management and low signaling load requirements. We have then proposed the cluster-based access control (CBAC) mechanism to mitigate D2D-to-cellular interference and enhance network throughput. Specifically, in the proposed mechanism, each SBS firstly forms a cluster of SUEs and DUEs, and selects qualified BWPs. Then, it jointly performs channel allocation and transmission power assignment to its SUEs or DUEs based on the estimated maximum D2D-to-cellular interference. Simulation results have proved that the proposed CBAC mechanism can provide higher network throughput as well as total throughput obtained by MUEs, SUEs, and DUEs (via D2D communications). There 


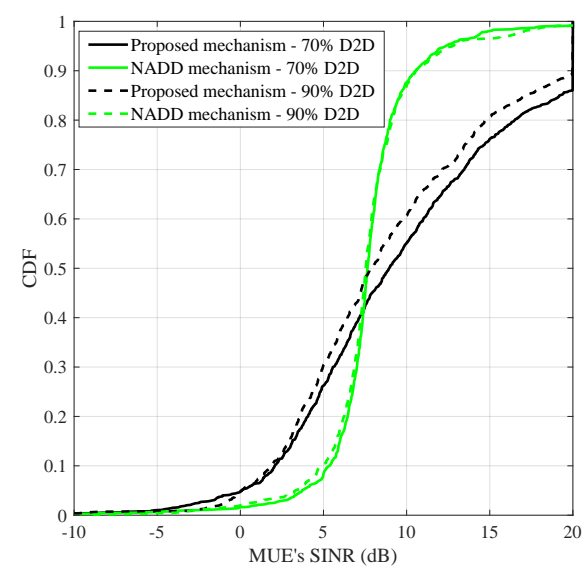

(a)

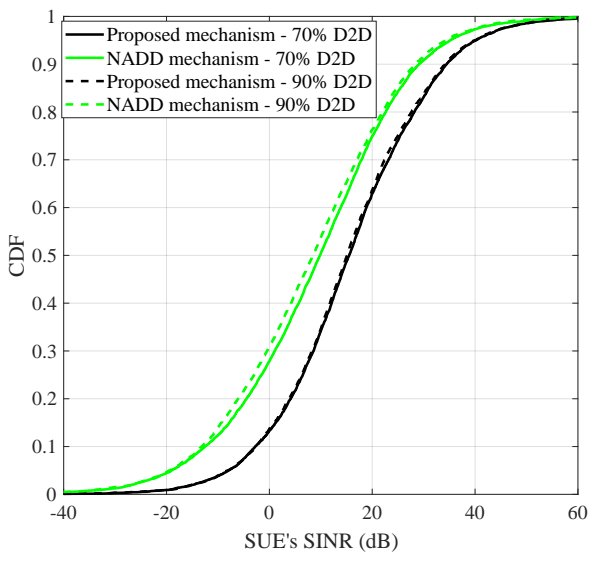

(b)

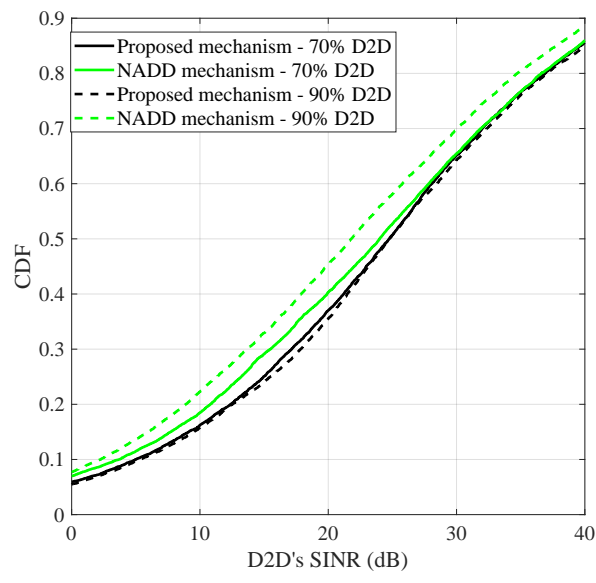

$(c)$

Fig. 4. (a) CDF of MUE's SINR, (b) CDF of SUE's SINR, (c) CDF of D2D's SINR.

are still open research issues for future research in resource management for heterogeneous cellular D2D communication networks with dense device deployment such as the cooperative access control between the MBS and SBSs, or a distributed transmission power optimization and interference mitigation problem.

\section{ACKNOWLEDGMENT}

This work has been supported by Vietnam National University, Hanoi (VNU), under Project No. QG.19.24.

\section{REFERENCES}

[1] W. Cao, G. Feng, S. Qin and M. Yan, "Cellular Offloading in Heterogeneous Mobile Networks With D2D Communication Assistance", IEEE Transactions on Vehicular Technology, vol. 66, pp. 4245-4255, Aug. 2016.

[2] Y. Niu et al., "Exploiting Device-to-Device Communications to Enhance Spatial Reuse for Popular Content Downloading in Directional mmWave Small Cells", IEEE Transaction on Vehicular Technology, vol. 65, pp. 5538-5550, Aug. 2015.

[3] W. Lee, J. Kim, and S. Choi, "New D2D Peer Discovery Scheme based on Spatial Correlation of Wireless Channel", IEEE Transaction on Vehicular Technology, vol. 66, pp. 10120-10125, Feb. 2016.

[4] M. Hicham, N. Abghour, and M. Ouzzif, "Device-To-Device (D2D) Communication Under LTE-Advanced Networks", International Journal of Wireless \& Mobile Networks, vol. 8, pp. 11-22, Feb. 2016.

[5] J. Huang et al., "Modeling and Analysis on Access Control for Deviceto-Device Communications in Cellular Network: A Network Calculus Based Approach", IEEE Transactions on Vehicular Technology, vol. 65, pp. 1615-1626, Mar. 2016.

[6] P. Mach, Z. Becvar, and T. Vanek, "In-band Device-to-Device Communication in OFDMA Cellular Networks: A Survey and Challenges", IEEE Communications Surveys \& Tutorials, vol. 17, pp. 1885-1922, Jun. 2015.

[7] C. Yu, K. Doppler, C. B. Ribeiro, and O. Tirkkonen, "Resource Sharing Optimization for Device-to-Device Communication Underlaying Cellular Networks," IEEE Transactions on Wireless Communications, vol. 10, no. 8, pp. 2752-2763, Aug. 2011.

[8] A. Bhardwaj and S. Agnihotri,, "Energy- and Spectral-Efficiency TradeOff for D2D-Multicasts in Underlay Cellular Networks", IEEE Wireless Communications Letters, vol. 7, pp. 546-549, Aug. 2018.

[9] G. A. Safdar, M. Ur-Rehman, M. Muhammad, M. A. Imran, and R. Tafazolli, "Interference Mitigation in D2D Communication Underlaying LTEA Network", IEEE Access, vol. 4, pp. 7967-7987, Oct. 2016.
[10] N. Lee, X. Lin, J. G. Andrews, and R. W. Heath, "Power Control for D2D Underlaid Cellular Networks: Modeling, Algorithms and Analysis", IEEE Journal on Selected Areas in Communications, vol. 33, pp. 1-13, Jan. 2015.

[11] W. Lee, T. Ban and B. C. Jung, "Distributed Transmit Power Optimization for Device-to-Device Communications Underlying Cellular Networks", IEEE Access, vol. 7, pp. 87617-87633, Jul. 2019.

[12] P. Gandotra and R. K. Jha, "Device-to-Device Communication in Cellular Networks: A Survey", Journal of Network and Computer Applications, vol. 71, pp. 99-117, Aug. 2016.

[13] F. Berggren and B. M. Popović, "Primary Synchronization Signal for D2D Communications in LTE-Advanced", IEEE Communications Letters, vol. 19, pp. 1241-1244, Jul. 2015.

[14] S. Cicalò and V. Tralli, "QoS-Aware Admission Control and Resource Allocation for D2D Communications Underlaying Cellular Networks", IEEE Transactions on Wireless Communications, vol. 17, pp. 5256-5269, Aug. 2018.

[15] D. Feng et al., "Device-to-Device Communications in Cellular Networks", IEEE Communications Magazine, vol. 52, pp. 49-55, May 2014.

[16] S. Lv, C. Xing, Z. Zhang and K. Long, "Guard Zone Based Interference Management for D2D-Aided Underlaying Cellular Networks", IEEE Transactions on Vehicular Technology, vol. 66, pp. 5466-5471, Oct. 2016.

[17] S. Liu, Y. Wu, L. Li, X. Liu and W. Xu, "A Two-stage Energyefficient Approach for Joint Power Control and Channel Allocation in D2D Communications", IEEE Access, vol: 7, pp: 16940-16951, Jan. 2019.

[18] Y. Yuan, T. Yang, H. Feng and B. Hu, "An Iterative MatchingStackelberg Game Model for Channel-power Allocation in D2D Underlaid Cellular Networks", IEEE Transactions on Wireless Communications, vol: 7, pp: 7456-7471, Sep. 2018.

[19] S. Yang, L. Wang, J. Huang and A. Tsai, "Network-assisted Devicedecided Channel Selection and Power Control for Multi-pair Device-toDevice (D2D) Communications in Heterogeneous Networks", in IEEE Wireless Communications and Networking Conference, Nov. 2014.

[20] B. Zhou, H. Hu, S. Huang and H. Chen, "Intracluster Device-to-Device Relay Algorithm with Optimal Resource Utilization", IEEE Transactions on Vehicular Technology, vol. 62, pp. 2315-2326, Jan. 2013.

[21] J. Ding, L. Jiang and C. He, "Energy-Efficient Power Control for Underlaying D2D Communication with Channel Uncertainty: User-Centric versus Network-Centric", Journal of Communications and Networks, vol. 18, pp. 589-599, Aug. 2016.

[22] H. Xu, W. Xu, Z. Yang, J. Shi and M. Chen, "Pilot Reuse among D2D Users in D2D Underlaid Massive MIMO Systems", IEEE Transactions on Vehicular Technology, vol. 67, pp. 467-482, Jul. 2017. 
[23] H. Sun, M. Wildemeersch, M. Sheng and T. Q. S. Quek, "D2D Enhanced Heterogeneous Cellular Networks with Dynamic TDD”, IEEE Transactions on Wireless Communications, vol. 14, pp. 4204-4218, Mar. 2015.

[24] H. Tang, Z. Ding and B. C. Levy, "Enabling D2D Communications through Neighbor Discovery in LTE Cellular Networks", IEEE Transac- tions on Signal Processing, vol.62, pp. 5157-5170, Aug. 2014.

[25] G. Liu, W. Feng, Z. Han and W. Jiang, "Performance Analysis and Optimization of Cooperative Full-Duplex D2D Communication Underlaying Cellular Networks", IEEE Transactions on Wireless Communications, vol.18, pp. 5113-5127, Aug. 2019. 